\title{
Challenges in the treatment of West syndrome
}

\author{
András Fogarasi*, Fruzsina Erdélyi, Zsuzsa Siegler, Márta Hegyi, Rita Jakus, Zsuzsa Gyorsok, \\ Tímea Bodó and Bence Ambrus
}

Department of Neurology, Bethesda Children's Hospital, Budapest, Hungary

Received: June 18, 2018; Published: July 03, 2018

*Corresponding author: András Fogarasi, Neurology Department, Bethesda Children’s Hospital, Budapest, Hungary

Abbreviations: ACTH: Adrenocorticotropic Hormon; UKISS: United Kingdom Infantile Spasms Study

\section{Introduction}

West syndrome is a rare and severe infant epileptic encephalopathy, covering $25-30 \%$ of infantile epilepsies. It's incidence is 2-3.5/ 10000 live births. The clinical picture usually starts with infantile spasms, which can occur in clusters and is often associated with developmental arrest or regression. In $75 \%$ of the cases the interictal EEG shows hypsarrhythmia, which is chaotic, high amplitude, slow wave activity. The underlying diseases can be a variety of different neurological etiologies, such as metabolic, structural developmental, perinatal lesions, genetic or neurocutan disorders. Despite the widening scale of diagnostic possibilities, detection of the underlying disease is unsuccessful in case of at least a quarter of the patients [1]. Early diagnosis and adequate therapy are key factors in achieving better outcomes [2]. Treatment of this clinical picture is still one of the unresolved questions of pediatric epileptology [3]. There is consensus on using adrenocorticotropic hormon (ACTH), steroid and vigabatrin as first line, standard therapy, but the dosage and the duration of such treatments are still a matter of debate. More recent studies recommend using combined vigabatrin and ACTH therapy, showing to achieve, in relatively short time, the highest outcome ratio of patients without spasms [4]. In our current study, we reviewed the outcomes resulting from various treatments applied at our institution and compared it with the available recommendations given in the relevant literature. Based on these findings, we have compiled a comprehensive guideline of therapy, aimed at optimizing care of patients with West syndrome.

\section{Methods Patient Selection}

In this current study we reviewed the cases of patients treated with West syndrome in our Neurology Department between 20132015, in Bethesda Children's Hospital, Hungary. Children selected for the study were diagnosed with West syndrome showed infantile spasms and hypsarrythmia on their EEG register and later attended our care system for at least one year. Altogether 35 children were selected into the study, 16 girls and 19 boys. Patients were divided into two groups, based on the underlying disease. Symptomatic group included those patients, where the diagnostic evaluation found any abnormalities possibly responsible for the epilepsy. The idiopathic group held those cases where all available diagnostic tests gave negative results, so no underlying disease was detected. Priour to our current research, no first line treatment had been established in our hospital for West syndrome. With the personal clinical experiences and the international recommendations we usually started the treatment with vigabatrin $(100-150 \mathrm{mg} / \mathrm{kg}$, reaching the doses within 3-4 days). In resistant cases we either increased the dosage or added on nitrazepam $(0,3-0,5 \mathrm{mg} / \mathrm{kg})$. If we still could not reach a spasm free state, intramuscular ACTH therapy or other types of antiepileptic drugs were given. The patients were told spasm free if they were seizure free for at least 3 months, spasms reoccurring after this period were considered as relapses.

\section{Results}

In our study t epilepsy started at age of 6 months (7 weeks- 2 years) and the diagnosis on average was made in 20 days after the occurrence of the spasms. 12/35 cases patients had different types of seizures before West syndrome evolved, and 9 kids already took antiepileptic medication. Three kids had to be excluded from the etiologic classification, because no brain MRI was performed. The symptomatic group held most of the children, 28/32. Underlying etiology was perinatal injury in 11 cases (brain MRI test showed intraventricular hemorrhage in 9 cases, stroke in 1 case and also severe periventricular leukomalacy in 1 case). Different diagnosis was found in the other 17 symptomatic cases, including lissencephaly ( 2 cases), polymicrogyria ( 2 cases), tuberous sclerosis ( 2 cases), HSV encephalitis (1 case) and genetic origin (3 cases, CDKL 5 mutation, 5q14.3q15 microdeletion syndrome, 3p12.3- 
3p12.2 region duplication). We found 7 cryptogenic cases, where brain MRI showed atypical picture (delayed myelinisation or brain atrophy), but the overall syndromatologyc classification was not obvious. Only 4/32 patients were classified in the idiopthic group.

During the follow-up of these patients we found that three idiopathic cases responded well to the first line vigabatrin therapy, reaching seizure freedom and only one idiopathic patient needed ACTH injections. In symptomatic cases, however, many therapeutic changes had to be made to reach seizure freedom or better seizure control. In 22/28 cases after several unsuccessful therapeutic attempt, ACTH injections finally brought the expected effect. Altogther 12/35 patients reached seizure freedom. All the idiopathic cases became free of spasms, unlike the symptomatic patients, where during the follow-up only 6 out of 28 children reached absolute seizure freedom. In 14/35 cases spasms and hypsarrythmia resolved, but after an average of 11 months (5 -18 months) other types of epileptic disorders developed. Various focal epilepsies (12 cases) and Lennox-Gastaut syndrome ( 2 cases) were observed, these patients needed long term antiepileptic medication. In case of the nine therapy resistant patients, spasms unfortunately never ceased. Amongst them, seven have experienced relapse after a period of temporary seizure freedom ( 4 to 18 months), while in two cases children did not experience a spasm free period of up to 3 months.

\section{Discussion}

Based on our study we cannot make absolute conclusions on the effectiveness of the medications used in the different etiologic groups, because of the small number of participants. We aimed at comparing our results with the recently published international literature to find correlations and create a management protocol for patients with West syndrome. We found that the idiopathic group responded well to vigabatrin therapy, experiencing seizure freedom in most cases. Numerous studies concluded similarly, that idiopathic etiology usually indicate a better response to oral medication then in symptomatic etiology [2,5] (Figures 1 \& 2). However, the United Kingdom Infantile Spasms Study (UKISS) found that even in the idiopathic cases the use of ACTH therapy is more beneficial, because a better cognitive outcome was seen in long term follow-up [6]. In the symptomatic group only ACTH therapy brought seizure freedom or better spasm control in a higher rate. Treatment strategy applied in our hospital for the symptomatic patients caused a delay in using the most effective ACTH therapy, even weeks or months went during the management of other less effective medications.

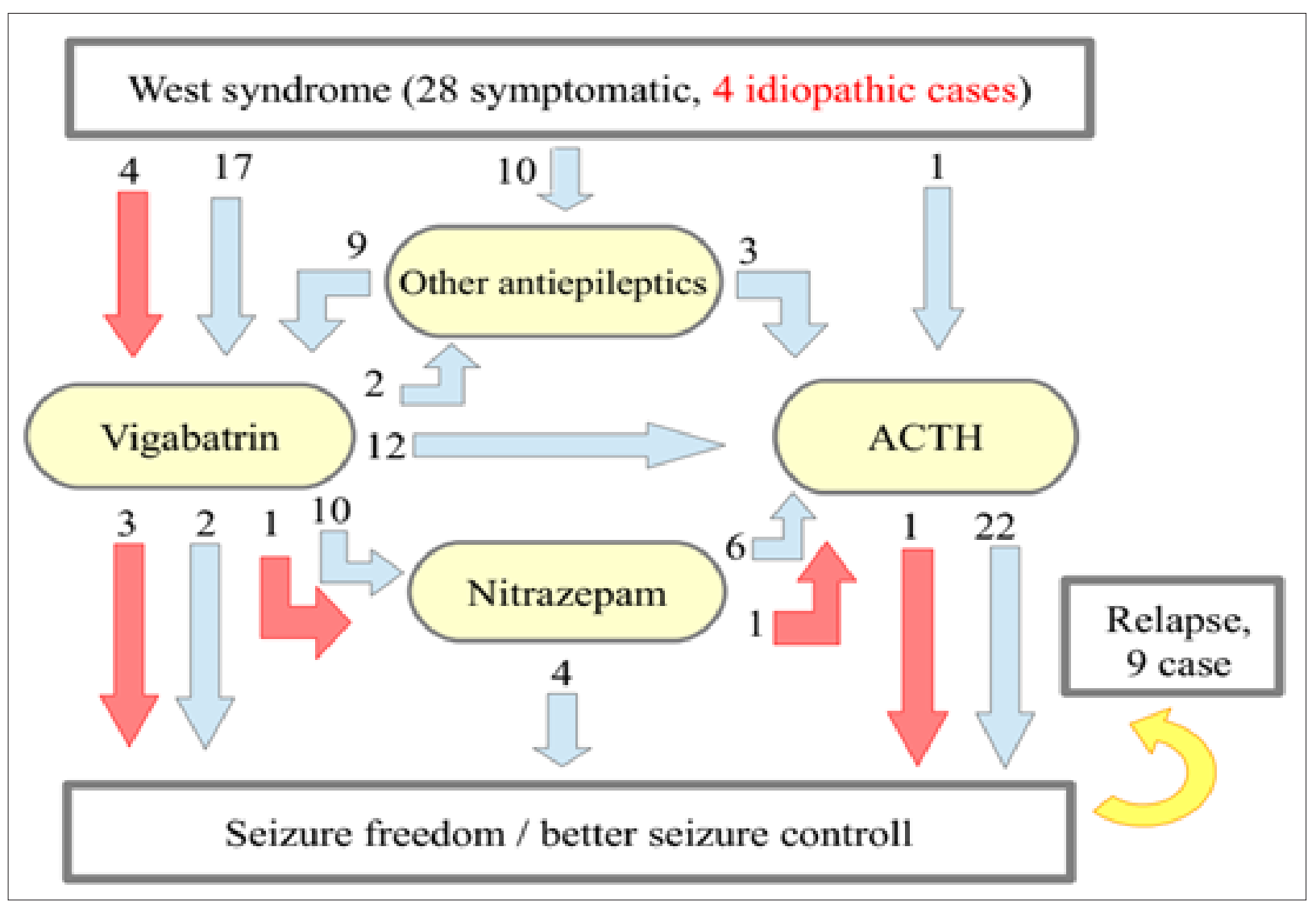

Figure 1: Therapeutic patient journeys of 32 infants with West syndrome. 


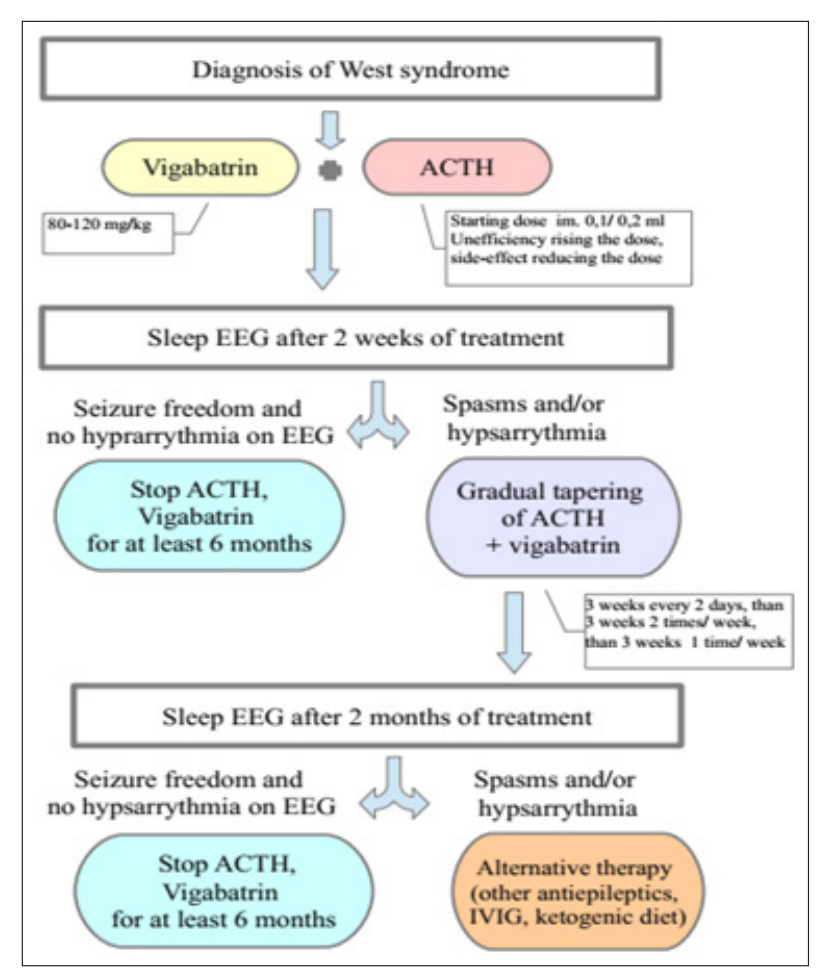

Figure 2: Management protocoll of infants with new-onset West syndrome.

Additionally, we must highlight what O' Callaghan and his colleagues' extended study suggests, that the combination of ACTH and vigabatrin was found to be the most effective, therefore combination therapy should be preferred in West syndrome [4]. Patients with known tuberous sclerosis are an exception for this recommendation, where the first line therapy is vigabatrin monotherapy worldwide [1,3]. During the medical treatment, frequent neurology follow-up visits and control of sleep EEG are recommended. It is important in the future to use objective scales to measure psychomotor development. We created our own management protocol according to the international recommendations and the experience of our recent study. Using a management protocol can help to optimize the start of adequate therapy as early as possible and the planning of the upcoming follow-ups.

In this protocol treatment is started with combination therapy (ACTH injection, plus vigabatrin) and taper the dosage if needed. The first sleep EEG should be controlled is due after two weeks of therapy. Subsequently, if the child is seizure free and the EEG shows no hypsarrythmia, we terminate the ACTH therapy and continue with vigabatrin monotherapy for 6 months. If total seizure freedom was not reached or the EEG still shows hypsarrythmia, ACTH therapy is tapered gradually and keep the vigabatrin therapy on. In case of no improvement with the first line combination therapy, alternative antiepileptic medication is given, for example topiramate or ketogenic diet has beneficial efficacy [3]. Next visit and EEG control is scheduled within 2 months.

\section{Conclusion}

Early recognition and urgent standard first-line treatment is of the highest importance in the treatment of West syndrome. We hope that an upto date management protocol will help to optimize the patient journey in the treatment of this age-related, rare and severe disease.

\section{References}

1. Nelson Gary Rex (2015) “Management of infantile spasms." Translational pediatrics 4(4): 260-270.

2. O'Callaghan, Finbar JK, Lux AL, Darke K, Edwards SW, et al. (2011) “The effect of lead time to treatment and of age of onset on developmental outcome at 4 years in infantile spasms: evidence from the United Kingdom Infantile Spasms Study." Epilepsia 52(7): 1359-1364.

3. Iyer Anand, Richard Appleton (2016) "Improving outcomes in infantile spasms: role of pharmacotherapy." Pediatric Drugs 18(5): 357-366.

4. O'Callaghan, Finbar JK, Edwards SW, Alber FD, Hancock E, et al. (2017) Safety and effectiveness of hormonal treatment versus hormonal treatment with vigabatrin for infantile spasms (ICISS): a randomised, multicentre, open-label trial." The Lancet Neurology 16(1): 33-42.

5. Jones Kevin, Go C, Boyd J, Ochi A, McCoy B, et al. (2015) "Vigabatrin as first-line treatment for infantile spasms not related to tuberous sclerosis complex." Pediatric neurology 53(2): 141-145.

6. Lux Andrew L, Edwards SW, Hancock E, Johnson AL, Kennedy CR, et al. (2005) "The United Kingdom Infantile Spasms Study (UKISS) comparing hormone treatment with vigabatrin on developmental and epilepsy outcomes to age 14 months: a multicentre randomised trial." The Lancet Neurology 4(11): 712-717. 
(c) (1) This work is licensed under Creative Submission Link: https://biomedres.us/submit-manuscript.php

Assets of Publishing with us
RESEARCHES $\quad \begin{aligned} & \text { Global archiving of articles } \\ & \text { - Immediate, unrestricted online access }\end{aligned}$

